\title{
Agent-based modeling of teacher interaction within a repository of digital objects
}

\author{
Evgeny Patarakin ${ }^{1 *}$, Svetlana Vachkova $^{1}$, and Vasiliy Burov ${ }^{1}$ \\ ${ }^{1}$ Moscow City University, Institute of System Projects, Moscow, Russia
}

\begin{abstract}
This article presents generalized model of collaborative actions, during which participants create, modify, and estimate digital objects. Such activities can be observed in numerous network communities. A prominent example is the repository of lesson scenarios of Moscow Electronic School (MES). The combination of methods of agent-based modeling and network analysis is used in the work. Using NetLogo environment in the frames of the model, an artificial community has been developed, where teachersagents interact with scenarios-agents. Teacher-agent determines whether there are potential scenarios in his environment to be contacted with. If such scenarios are available, then the agent selects the nearest one and makes a step towards it. If the scenario has been opened by one of the teachers, then this is already an author's scenario and the teacher-agent takes an action to reuse it. Variants of the reuse can be preset so that to correspond to the actions allowable in the environment of MES repository for learning scenarios: review, addition to bookmarks, running the scenario, downloading, using in home assignments. All these actions of teachers regarding scenarios are logged, then the log records are transformed into bipartite graph. The experiments demonstrate that while the area of participant scenarios is expanded, not only the general number of links among participants increases but also large networks of participants are subdivided into smaller and densely interconnected groups. One of the control trends of participant activities is in the use of multiagent-based modeling as a tool of collective reflection of teachers cooperating on the basis of MES.
\end{abstract}

Keywords: digital repository, digital object, network analysis, agent-based modeling.

\section{Introduction}

Creation and use of digital educational content by teachers and students on the basis of educational communities is nowadays an inherent part of pedagogical activities. It is performed both on the basis of educational resources and on the basis of resources, which are not directly educational but support work of topical communities related with education activities $[1,2]$.

\footnotetext{
* Corresponding author: patarakined@mgpu.ru
} 
With all variety of collaborative network activities, the actions can be presented in the form of a single flowchart, which allows to analyze and to compare the activities of participants. The most well-known Russian system is Moscow Electronic School (hereinafter referred to as MES), where several roles exist for users. The actors of the activities are teachers, students, parents, publishers: all persons who perform any actions with the objects; an object is created by a participant-author, the author assigns properties to the object, gives name, describes the object, refers it to school subject and level of education.

All history of actions by the participants is logged, the log is available for analysis of actions of any participant and variation of each object. The logged history can be presented as a record containing the following mandatory elements:

1. Actor (agent) of the activities. In general case, the agent ID is known.

2. Object of the activities: text, scheme, presentation, lesson scenario, etc.

3. Type of activities: action applied by the subject to the object.

4. Time of activities: record when the action was performed.

General approach to transform the history of actions by the subjects to the objects of activities is described in publication devoted to learning analytics, in which the history was transformed into relation graphs: bipartite graphs with subjects and objects of the activities, and unipartite graphs of interrelations among the participants $[3,4]$.

\section{Methods}

Agent-based modeling is concentrated on individual participants in the system. This is its difference from more abstract method of system dynamics and discrete event method oriented at processes. In the agent-based modeling, the parameters of active agents-objects are preset and their behavior is defined. Models with preset variation of state, related with interaction of various agents having noncorrelated behavior, are multiagent-based [5-7]. Network analysis studies the issues of creation of links of various orientation among the objects and distribution of information determining actions of the objects [8-10]. Combination of methods of agent-based modeling and network analysis allows to simulate sufficiently precisely the situation in real network communities and to perform computing experiments revealing methods of efficient control action on community.

Computer simulation opens cardinally new opportunities of analysis of social processes for social sciences. The most obviously the new approach is determined by Epstein as generative or computational sociology, the objective of which is to grow phenomenon to be understood by researcher [11].

Modeling is generally related with analysis of complicated and complex systems with high amount of agents or nodes, that is, the content area of agent-based modeling intersects with the science of networks and complex systems [12-14]. Thus, the use of multiagent-based modeling in combination with network analysis seems to be promising $[15,16]$.

Various environments of agent-based modeling, systemized on the basis of specially developed typologies, are analyzed in [17]. Among the developed researching environments, the leading position is occupied by NetLogo.

Using the model in NetLogo environment, we wanted to obtain data and visualizations, which would assist to understand better the events and network structures occurring during interaction of numerous agents in MES environment.

\section{Discussion}

The artificial world created in the frames of multiagent model includes the field, where teachers-agents and scenarios-agents can move. Teachers are initially connected with 
schools. When the model is run, schools are preset initially and then teachers are found around them. There are some restrictions in the model, for instance, teachers cannot vary their belonging to school. In addition to belonging to school, the teacher is interconnected with one or several school subjects. Behavior of agents in the model is determined by rules. Teacher-agent determines whether there are balls-potential scenarios in his surrounding, which he could touch. If such balls are available, then the agent selects the nearest one and makes a step towards it. If the teacher touches the ball, then he applies an action to it. If this is the first touch, then the action is creation of scenario, herewith the teacher marks it with the tag of school subject taught by him. As a consequence, the ball varies its position in the screen and the color, and the record is logged that the teacher-agent performed actions to the ball-agent, the action was performed at certain time. One of the trends of development of the models will be related with the analysis of agent behavior dynamics.

If the ball scenario has already been opened by a teacher, then this is an author's scenario and the teacher-agent reuses it: the ball varies its position in the screen, and the record is logged that the teacher-agent reused the ball-agent, which took place at certain time.

During actions by teachers-agents with scenarios-agents the results are reflected in histogram of distribution of scenarios properties: the number of potential, created and reused scenarios.

After completion of preset number of actions with objects by participants, the system activity is terminated and a researcher can obtain the graph of interrelations among the participants during their joint actions with the objects.

Several parameters are active in the system, which determine behavior of the participants and which can be controlled by a researcher. For the situation with about 1,000 agents and 2,370 potential scenarios, the number of cycles was experimentally selected equaling to 15 . Herewith, there was sufficiently high number of scenarios, with which no teachers-agents interacted (Fig. 1). 


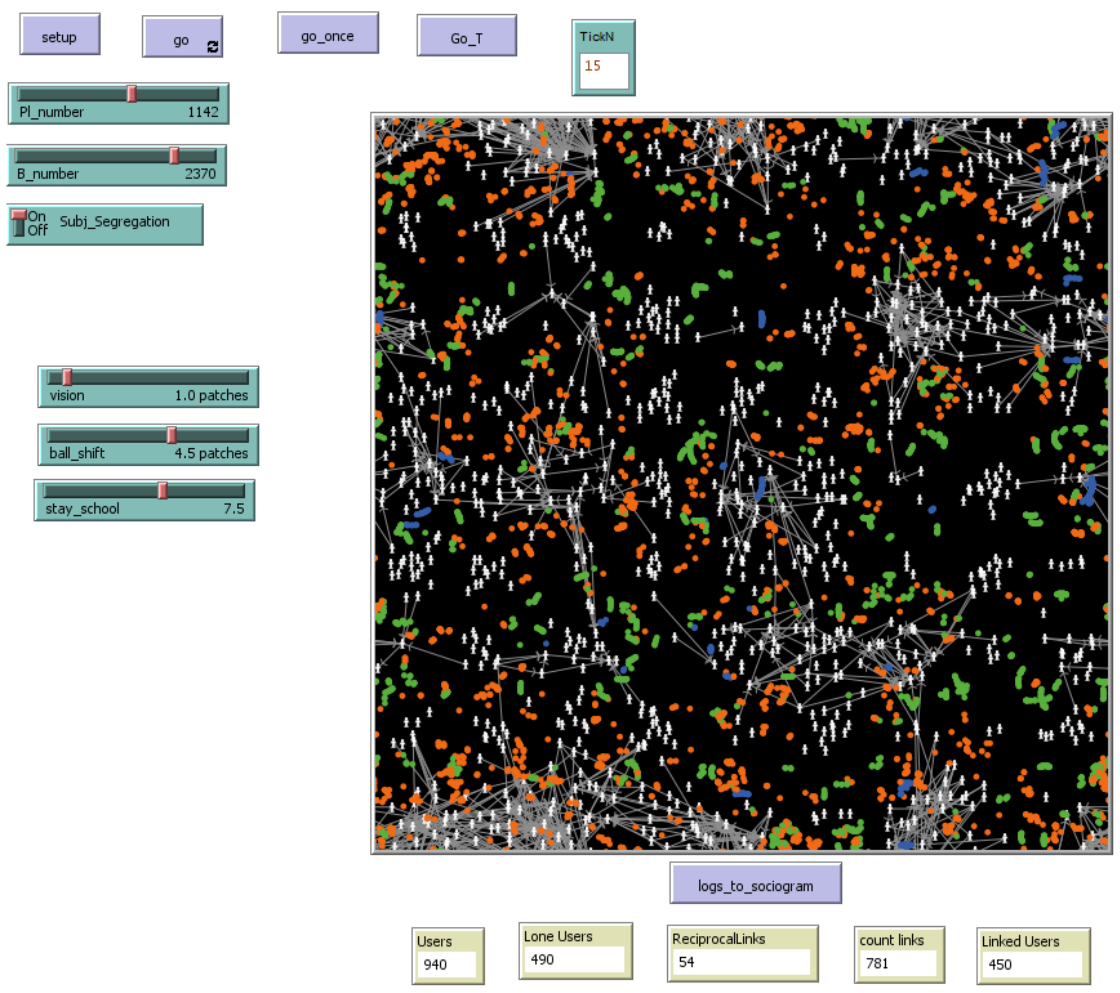

Fig. 1. Model interface with control buttons and sliders.

A fragment of interface, located in the model to the right from the area of simulation and reuse of digital content, is illustrated in Figure 2.

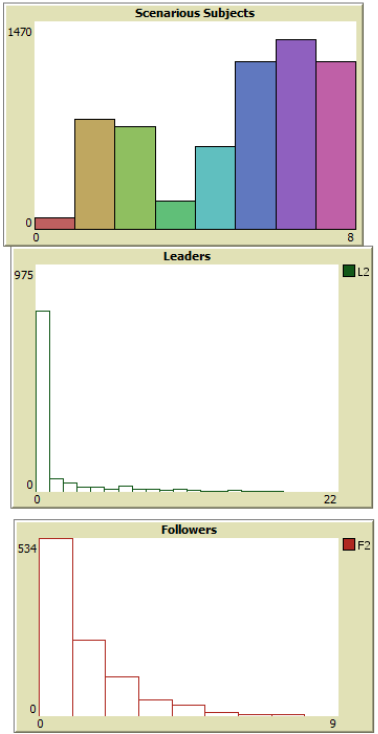

Fig. 2. Interface: visualization of data distribution. 
One of the tasks in this project was comparison of the results of simulation of teachers' actions in artificial model with those based on real teachers' actions in MES repository. Analysis and visualization of actions of real community and records about actions of agents within the artificial community were recorded and processed in the $\mathrm{R}$ language environment (Fig. 3).

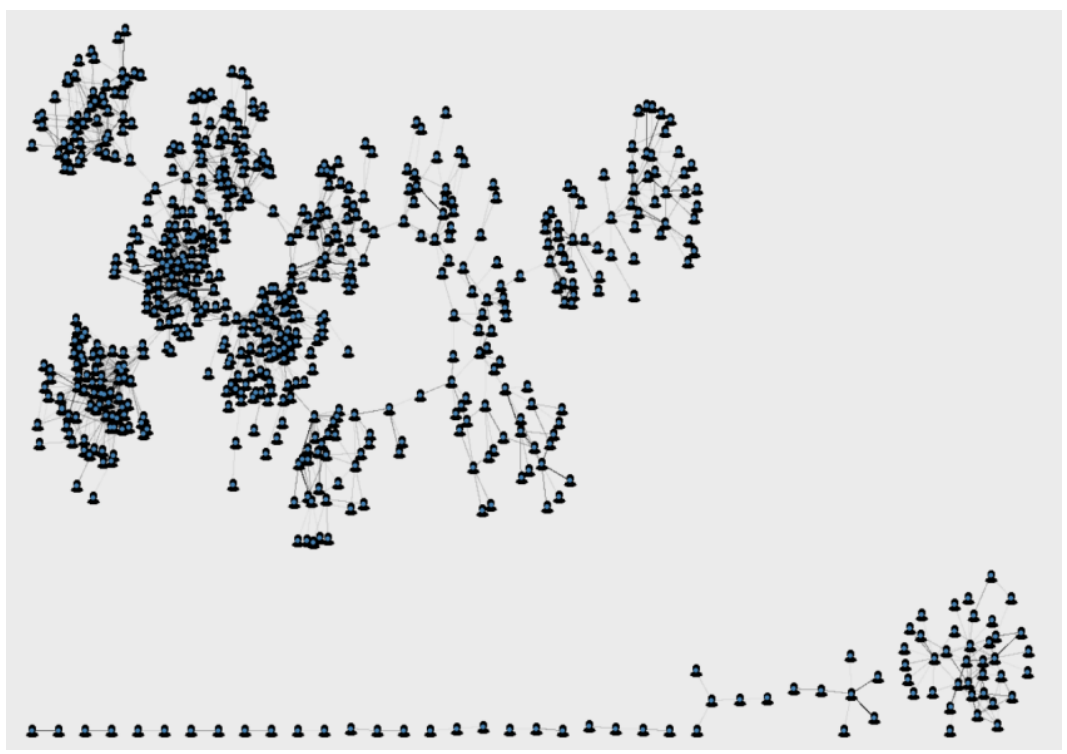

Fig. 3. Visualization of relations among the agents on the basis of data obtained by model of virtual community.

General flowchart of the experiments. A control action is selected, which will be estimated in the experiment, as well as the model parameters, which can be controlled by a user; the simulation of joint action of a set of agents is run. After completion of actions of the artificial community, the resulted traces of the actions are stored in the form of graphs and histograms in separate datasheet for further analysis. Then the data are processed, reduced to the form corresponding to the form of datasheet from the environment of MES network community, and visualized. The obtained results are compared.

The second trend of analysis was related with the influence of subdivision of participants into groups with different rules of behavior: those, who could use any available scenarios, and those, who could use the scenarios marked by tags of the subjects taught by the agent. Initially we expected that such impact on behavior of participants could lead to formation of interschool subject groups.

In artificial communities, several types of agent behavior can be tuned by toggling the sliders into On/Off position. For instance, if we set Self_Use into On (true) position, then the agent refers equally to all subjects in the vision area. This strategy is allowed in MES, when a teacher can estimate own scenarios, add them to bookmarks, download. If we select Off, then the teacher can reuse only scenarios created by other participants.

Another opportunity is to preset behavior type by toggling subject flag. If this tag is deactivated, then the teacher-agent equally interacts with all objects in the vision area. At activated flag, the teacher interacts only with objects, which are marked by the same tags of subject taught by him. 


\section{Results}

The experiments demonstrate that the number of single participants (silent observers) in the artificial system is not so high as in natural system; however, it is above $20 \%$.

We believed that expansion of the area of visible digital objects for teachers- agents would lead to equalization of network indicators, combination of all participants into one giant component, combining all participants. However, the results demonstrate that while the common number of links among the participants increases with the expansion of the vision area, large components are subdivided into smaller and densely interconnected groups. Therefore, we observe the results of control action on behavior of participants, quite often this contradicts with the expectations as a consequence of the control actions.

The control actions used in the experiments have allowed to set the following questions:

1. What will occur if we suggest to the teachers to pay more attention to the scenarios created by the colleagues from their schools? Would this lead to formation of higher number of steady groups of participants combined by reciprocal links, who use projects created by their school colleagues?

2. What will occur if we restrict the rate of propagation of learning scenarios? What will occur if we increase the rate of propagation of materials and the results created by teachers of one school become visible for other schools?

What will occur if scenarios, rigidly connected to the taught subjects, are offered to the teachers for analysis, estimation, copying? Will this result in formation of subject interschool groups?

\section{Conclusion}

Control of activities of participants in MES network community on the basis of modeling of MES development in artificial community in multiagent-based modeling environment assumes several possible directions:

Adding changes into MES repository system design. Using smart system, which adjusts the participant's opportunities to the actions already carried out and to the information about the participant available in the system. Formation of information flows is important, where personal data about the participant are accounted (subjects of interest, age of studied pupils, etc.) on the basis of initial information about the participant and digital traces.

Subdivision of active participants into groups, where they interact more among themselves: on the basis of common subjects, common schools, etc. Such subdivision leads to formation of additional links among the participants and strengthens the system of mutual actions in total. It would be reasonable to add the toolset to the MES repository design, which would support free formation of groups by interests and by any grounds: any ground for selforganization into groups for joint activities would promote formation of additional links and would increase total social capital within the MES repository.

Using multiagent-based modeling as a tool for collective reflection of teachers cooperating on the basis of MES.

\section{References}

1. V. Franzoni, S. Tasso, S. Pallottelli, D. Perri, Sharing Linkable Learning Objects with the Use of Metadata and a Taxonomy Assistant for Categorization, in Proceedings of the 19th International Conference Computational Science and Its Applications ICCSA 2019, Lecture Notes in Computer Science, vol. 11620, 1-4 July 2019, Saint Petersburg, Russia (2019). https://doi.org/10.1007/978-3-030-24296-1_28 
2. M. Talbot, K. Geldreich, J. Sommer, P. Hubwieser, Re-use of programming patterns or problem solving? Representation of scratch programs by TGraphs to support static code analysis, in Proceedings of the 15 th Workshop on Primary and Secondary Computing Education, WiPSCE'20, 28-30 October 2020, Virtual Event, Germany (2020). https://doi.org/10.1145/3421590.3421604

3. E. Patarakin, V. Burov, Using of Automatically and Semi-automatically Generated Diagrams in Educational Practice, in Proceedings of the $6^{\text {th }}$ International Conference Electronic Governance and Open Society: Challenges in Eurasia, EGOSE 2019, Communications in Computer and Information Science, vol 1135, 13-14 November 2019, Saint Petersburg, Russia (2020). https://doi.org/10.1007/978-3-030-39296-3_23

4. E.D. Patarakin, Wikigrams-Based Social Inquiry, in Digital Tools and Solutions for Inquiry-Based STEM Learning, 112-138 (IGI Global, Hershey, 2017). https://doi.org/10.4018/978-1-5225-2525-7.ch005

5. N. Gilbert, Agent-Based Models (Sage Publications, Newbury Park, 2007). https://dx.doi.org/10.4135/9781412983259

6. Y. Shoham, K. Leyton-Brown, Multiagent Systems: Algorithmic, Game-Theoretic, and Logical Foundations (Cambridge University Press, Cambridge, 2008)

7. F. Squazzoni, Agent-Based Computational Sociology (John Wiley \& Sons, New Jersey, 2012)

8. A.-L. Barabási, Network Science (Cambridge University Press, Cambridge, 2016)

9. P.S. Smith, P.J. Trygstad, M.L. Hayes, International Journal of Leadership in Education, 21, 95-103 (2018). https://doi.org/10.1080/13603124.2016.1195016

10. N.H.D. Terblanche, E.D. Erasmus, SA Journal of Industrial Psychology, 44, 1-10 (2018). https://doi.org/10.4102/sajip.v44i0.1548

11. J.M. Epstein, Generative Social Science: Studies in Agent-Based Computational Modeling (Princeton University Press, Princeton, 2007)

12. R. De Caux, An agent-based approach to modelling long-term systemic risk in networks of interacting banks, $\mathrm{PhD}$ Thesis (University of Southampton, Southampton, 2017)

13. H. Sayama, C. Cramer, L. Sheetz, S. Uzzo, Complicity: An International Journal of Complexity and Education, 14(2), 104-115 (2017). https://doi.org/10.29173/cmplct29339

14. D. Secchi, M. Neumann (Eds.), Agent-Based Simulation of Organizational Behavior (Springer International Publishing, Cham, 2016)

15. T. Meyer, S. Upton, M. McDonald, C. Bouwens, Investigating Social Network Analysis Methods for Identifying Emergent Behaviors in Agent-Based Models (Naval Postgraduate School, Monterey, 2018). Accessed on: December 20, 2020. [Online]. Available: http://hdl.handle.net/10945/60822

16. G.M. Reséndiz-Benhumea, T. Froese, G. Ramos-Fernández, S.E. Smith-Aguilar, Applying Social Network Analysis to Agent-Based Models: A Case Study of Task Allocation in Swarm Robotics Inspired by Ant Foraging Behavior, in Artificial Life Conference Proceedings, 29 July - 2 August 2019, Newcastle, United Kingdom, 616623 (2019). https://doi.org/10.1162/isal_a_00229

17. K. Rakić, M. Rosić, I. Boljat, Tehnički vjesnik, 27, 1014-1020 (2020). https://doi.org/10.17559/TV-20190517110455 